\title{
Relation between the occurrence rate of ESF and the equatorial vertical plasma drift velocity at sunset derived from global observations
}

\author{
C. Stolle ${ }^{1}$, H. Lühr ${ }^{1}$, and B. G. Fejer ${ }^{2}$ \\ ${ }^{1}$ Deutsches GeoForschungsZentrum, Telegrafenberg, 14473 Potsdam, Germany \\ ${ }^{2}$ Center for Atmospheric and Space Science, Utah State University, Logan UT 84322, USA
}

Received: 18 June 2008 - Revised: 29 September 2008 - Accepted: 5 November 2008 - Published: 8 December 2008

\begin{abstract}
In this study, we investigate two global climatological data sets; the occurrence rate of Equatorial Spread$F$ (ESF), associated with equatorial plasma irregularities, at $\sim 400 \mathrm{~km}$ altitude obtained from CHAMP observations, and the evening equatorial vertical plasma drift, $v_{z}$, from ROCSAT-1 measurements. First, as retrieved for a solar flux level of F10.7 $=150$, the longitudinal variation of the two independently derived quantities correlates between $84 \%$ and 93\% in the seasons December solstice, equinox and June solstice. The highest correlation is found for the solstice seasons when $v_{z}$ is integrated over local time around the prereversal enhancement (PRE) and displaced $6^{\circ}$ towards east. The integrated $v_{z}$ is a suitable estimate of the ionospheric height at the time just after the PRE and the $6^{\circ}$ displacement is consistent with ESF eastward drift during $2 \mathrm{~h}$ which is assumed between creation and detection at satellite altitudes. Second, our analyses reveal a global threshold $v_{z}$ which is required to observe ESF at satellite altitudes. This threshold depends linearly on solar flux with correlations of $97 \%$. Both results bring global evidence on the linear relations between ESF and the vertical plasma drift which have been proven only by local observations so far.
\end{abstract}

This paper includes the first global map of the seasonal/longitudinal variation of the ESF occurrence rate over local time being valid for high solar flux years 2001-2004. The map reveals, e.g. a longitudinal dependence of the persistence of the plasma irregularities indicating that longitude dependent mechanisms other than the PRE determine the ESF lifetime.

Keywords. Ionosphere (Equatorial ionosphere; Ionospheric irregularities)

Correspondence to: C. Stolle

(stolle@gfz-potsdam.de)

\section{Introduction}

Over the last several decades the name Equatorial Spread- $F$ (ESF) has been used to designate equatorial plasma density irregularities over a large range of scale sizes which occur regularly in the low-latitude ionosphere after sunset. ESF events are believed to be generated in the bottom side Fregion when the ionosphere is unstable to the growth of the Rayleigh-Taylor (R-T) instability (e.g. Kelley, 1989). During their evolution they rise upward and increase in latitudinal extent (e.g. Whalen, 2000). Strong ESF events are associated with large scale plasma density depletions observed by rockets and satellites and with plasma bubbles detected by radar measurements, also known as plumes. They drift predominantly eastward with velocities of more than $100 \mathrm{~m} \mathrm{~s}^{-1}$ and are often tilted westward (see e.g. Sobral et al., 1999; Pimenta et al., 2003; Lin et al., 2005; Park et al., 2007; Yao and Makela, 2007; Makela and Kelley, 2003). Many climatological features of the ESF occurrence rates at specific locations in different longitude sectors have been revealed from the analyses of ground-based observations (see for example Fejer et al., 1999; Abdu et al., 2000; Sahai et al., 2004; Wiens et al., 2006). These studies have shown that the seasonal ESF occurrence is longitude dependent, and that the number of strong ESF decreases during periods of low solar flux activity. Global maps of ESF related occurrence rates were derived from satellite observations of local plasma depletions (e.g. Kil and Heelis, 1998; Huang et al., 2001; Palmroth et al., 2001; Burke et al., 2004; Park et al., 2005) or from total electron content observations at a ground-based GPS receiver network (Nishioka et al., 2008). Alternatively, Stolle et al. (2006) obtained the ESF distribution from magnetic signatures of the instabilities. All these observations revealed a typical seasonal/longitudinal (s/l) distribution of the ESF occurrence rate. The most striking feature is the very high rate in the Brazilian/Atlantic sector during December solstice

Published by Copernicus Publications on behalf of the European Geosciences Union. 
months. This sector is almost void of events during June solstice months. A more longitudinally uniform distribution is found around equinoxes.

Although the longitudinal distribution of occurrence rates of ESF are frequently observed, the reason of this typical feature is still a subject of research. The generation of ESF by the generalized R-T theory is described with a linear growth rate of:

$\gamma \approx \frac{\Sigma_{P}^{F}}{\Sigma_{P}^{F}+\Sigma_{P}^{E}}\left[\boldsymbol{v}_{z}-\frac{g}{v_{i n}}\right] \cdot \frac{\nabla n}{n_{0}}-R$

which includes gravitational and plasma density terms (Ossakow, 1981), as well as terms dealing with the effect of plasma drift (Ott, 1978), and conductivity distribution (Sul$\tan , 1996$ ). In Eq. (1), $\boldsymbol{v}_{z}=\boldsymbol{E} \times \boldsymbol{B} / B^{2}$ is the vertical component of the plasma drift related to the zonal component of the electric field $\boldsymbol{E}$ at the magnetic equator, $\boldsymbol{g}$ is the acceleration due to gravitation, $v_{i n}$ is the ion-neutral collision frequency, $n_{0}$ is the background electron density, $\Sigma_{P}^{F, E}$ are the flux tube integrated conductivities in the F- and E-region, respectively, and $R$ is the recombination rate. According to this equation, ESF evolution is strongly determined by the strength of the plasma density gradient. Ott (1978) emphasised the importance of the plasma drift component parallel/antiparallel to the density gradient which favors/reduces the probability for ESF onset. Furthermore, upward plasma drift near sunset lifts the ionosphere to higher altitudes where $v_{i n}$ is reduced, which additionally enhances $\gamma$. At this local time, the upward velocity amplitude usually increases sharply before it turns downward at night (e.g. Farley et al., 1986). This local plasma drift velocity peak is called the prereversal enhancement (PRE). The integrated E-region conductivity also contributes to the growth rate, and Tsunoda (1985) proposed that the alignment of the magnetic field with the sunset terminator plays an important role for its determination. Meridonal winds can also influence the hemispheric distribution of the plasma and the ESF occurrence (e.g. Kil and Heelis, 1998). McClure et al. (1998) proposed the importance of gravity waves to initiate the R-T instability process.

The close relations between the ESF occurrence and the amplitude of the vertical plasma drift and the layer height was shown empirically in several studies (e.g. Farley et al., 1970). Local evidence was given by Fejer et al. (1999) based on radar observations from the Peruvian Jicamarca Observatory who reported that the evolution of the unstable layer depends on the history of the evening vertical drift velocity. Mendillo et al. (2001) analysed GPS observations in the western part of South-America and proposed that the vertical plasma drift at sunset is the necessary and sufficient parameter in ESF forecast. Jayachandran et al. (1993) investigated HF Doppler radar and ionosonde measurements in India, and stated that the height of the F-layer, determined by the evening vertical plasma drift, is the deciding factor for ESF onset. They also stated that the R-T growth rate maximises at the time of peak height rather than at the time of peak velocity. Maruyama et al. (2002) proposed that inclined vortexlike $\boldsymbol{E} \times \boldsymbol{B}$ drift which creates upward drift in the lower Fregion (lifting the ionosphere) and downward drift in the upper ionosphere (steepening the vertical density gradient) is especially favorable for the gravitational R-T instability. Recently, Su et al. (2008) provided the global evidence of the relation between these two parameters. These authors compared the mean equatorial vertical plasma drift velocity between 18:00 LT and 19:00 LT with plasma depletion observations, both derived from ROSCAT-1 satellite measurements in different longitude sectors and found correlations coefficients up to 0.91 in December solstice. They were able to define the post-sunset $\mathbf{v}_{\mathbf{z}}$ as the "final indicator" for their statistical s/l ESF distribution.

A threshold velocity or, correspondingly, a threshold height of the ionosphere was proposed to produce positive R-T growth rates, and this threshold was found to vary with solar flux. Over Jicamarca, Fejer et al. (1999) reported a threshold plasma velocity for the generation of strong ESF of $\sim 50-60 \mathrm{~m} / \mathrm{s}$ for a solar flux index of 250 , and they found it to decrease to $\sim 5-10 \mathrm{~m} / \mathrm{s}$ for a solar flux index of 70 . Similar observations have been made in India by Jayachandran et al. (1993), who suggested increasing threshold velocity from 20 to $30 \mathrm{~m} / \mathrm{s}$ when the mean solar flux index increases from 70 to 120 . Manju et al. (2007) investigated ionosonde observations in Trivandrum, India and found a threshold ionospheric height for the generation of Spread- $F$ which was larger during solar maximum years than during solar minimum year.

In this paper, we compare two independent climatological data sets; the ESF occurrence rates derived from CHAMP satellite observations (Stolle et al., 2006) and the PRE vertical drift velocity derived from ROCSAT-1 satellite observations (Fejer et al., 2008). We make use of the full local time plasma drift description. This allows us to concentrate on the PRE drift velocity peak, as well as on the integrated PRE velocity, which provides an estimate of the evening ionospheric uplift. Our observations also provide the opportunity to investigate the linear dependence of the threshold velocity to generate ESF from our global view. Furthermore, we use the multi-year satellite data bases to look for seasonal and longitudinal differences in the response time of ESF occurrence to PRE actions, as well as for possible longitudinal dependencies of the ESF occurrence persistence. By this, we expect to contribute to the understanding of the validity of different ESF properties on global scale and in climatological sense, which were reported from local and time restricted observations so far.

The following section introduces the applied satellite data sets, the third section presents our observations, the fourth contains the discussion, and last one summerizes our results. 


\section{Data}

CHAMP (CHallanging Minisatellite Payload) was launched on 15 July 2000 into a near-circular orbit with an inclination of $87.3^{\circ}$ and an initial orbit altitude of $456 \mathrm{~km}$, which has decayed to about $400 \mathrm{~km}$ in 2004 (Reigber et al., 2002). Its orbital plane precesses through all local times every 4 months and through all longitudes at a fixed local time every $24 \mathrm{~h}$. The satellite carries, among other instruments, a fluxgate magnetometer which provides precise measurements of the Earth's magnetic field. Stolle et al. (2006) have shown that these high quality magnetic field observations contain signatures of equatorial plasma instabilities. For identifying them, all other contributions to the geomagnetic field have to be subtracted from the measurements, e.g. as by means of the POMME-3 model (Maus et al., 2006). POMME (Potsdam Magnetic Model of the Earth) comprises the main magnetic field, the litospheric magnetization and the effect of largesacle magentospheric currents. Orbital segments between $\pm 45^{\circ}$ geogr. latitude are selected and each segment is highpass filtered with a cut-off period of $30 \mathrm{~s}$ and the AC signal is rectified. The filtered time series are checked for peaks higher than $0.25 \mathrm{nT}$. Plasma irregularities are identified when several neighboring peaks are found within a $6^{\circ}$ latitudinal window. Only satellite observations during low magnetic activity $\left(K_{p}<3\right)$ have been considered since other magnetic field fluctuations may confuse the ESF detection algorithm during active periods. The occurrence rate was calculated as the number of orbital segments with ESF encounters over the sum of segments with or without ESF encounters, e.g. in a longitudinal bin. The climatological features such as seasonal/longitudinal and local time occurrence rate distributions derived from the multi-years magnetic survey (Stolle et al., 2006) compare very well with the global distribution of ESFs derived from plasma depletion observations (e.g. Kil and Heelis, 1998; Huang et al., 2001; Burke et al., 2004; Park et al., 2005). This confirms the reliability of magnetic measurements for detecting these F-region irregularities. Here, we make use of an ESF climatology that was derived from 4 years of CHAMP observations between 1 January 2001 and 31 December 2004 and between 18:00 and 02:00 LT. The mean solar flux level during these CHAMP observations was F10.7 $=149$. At the time of writing, CHAMP is still operational, and the present data set covers 8 years between 2000 and 2008.

Fejer et al. (2008) presented a climatological model of the equatorial F-region vertical plasma drift, which is based on measurements taken by the Ionospheric Plasma and Electrodynamic Probe Instrument (IPEI) on board the ROCSAT-1 satellite (Yeh et al., 1999). ROCSAT-1 was launched on 27 April 1999 into a circular orbit at an altitude of $600 \mathrm{~km}$ and with an inclination of $35^{\circ}$. The mission, which ended July 2005, sampled the dip equator at all local times in 25 days. IPEI provided continuous measurements between April 2000 and June 2004. The vertical drift observations which entered the model were obtained within a latitudinal range of $\pm 5^{\circ}$ about the dip equator. The model describes the equatorial F-region vertical plasma drift velocity during low magnetic activity $\left(K_{p}<3\right)$ and for 11 levels of solar flux from F10.7=100 to F10.7=200 in steps of 10 . Data are sorted into three seasonal bins which are December solstice (November-February), June solstice (May-August), and equinox (March-April, September-October). The longitudinal variation is specified at $15^{\circ}$ spacing between $-180^{\circ} \mathrm{E}$ and $+165^{\circ} \mathrm{E}$ derived from overlapping $30^{\circ}$ wide longitudinal bins. Local time is provided in $30 \mathrm{~min}$ steps derived from overlapping 1-h local time bins, except for the 17:0022:00 LT sector, where $30 \mathrm{~min}$ bins and $15 \mathrm{~min}$ resolution are used. Due to seasonal dependent data availability the model is most accurate for equinox and for December solstice. In the following, we will mainly use modelled plasma drift velocities derived for a solar flux level of F10.7 $=150$, which corresponds well with the mean solar flux level of nightside CHAMP observation between 2001 and 2004.

Figure 1 provides two examples of the local time variation of the vertical plasma drift velocity, $v_{z}$, as provided by the ROCSAT- 1 model at $-75^{\circ} \mathrm{E}$ and at $0^{\circ} \mathrm{E}$ separately for each season. The vertical plasma drifts are predominantly downward during night time and upward during the day with pre-noon maxima. The magnitude of the drifts decreases in the afternoon before they exhibit another positive maximum after sunset. Afterwards, the drift turns downward again. At few locations no PRE peak occurs like at $-75^{\circ} \mathrm{E}$ during June solstice (i.e. Fig. 1).

\section{Observations}

This paper investigates the effect of the plasma drift velocity during the prereversal enhancement on the occurrence probability of ESF. Therefore, we concentrated on two parameters which typically characterize the post-sunset plasma uplift: the peak value of the vertical plasma drift velocity during PRE, $v_{z}^{P}$, and the integrated vertical plasma drift velocity, $S_{\mathrm{PRE}}$, which is a measure of the uplift of the F-region plasma. We retrieved $v_{z}^{P}$ by searching for the highest drift value measured by ROCSAT-1 between 17:00 and 21:00 LT at each longitude for each of the three seasonal periods provided by the model. No typical PRE plasma drift pattern with a post-sunset local maxima is visible at the longitude bins $-90^{\circ},-75^{\circ}$, and $-60^{\circ} \mathrm{E}$ during June solstice. In those cases, we have chosen $v_{z}^{P}$ as the plasma drift value from the bin just before the time at which the plasma drift velocity decreases rapidly towards its night time values. For example, in the upper panel of Fig. 1, the value at 18:00 LT was chosen in case of June solstice.

The quantity $S_{\text {PRE }}$ is calculated by adding up the vertical drift values obtained at a local time node, $i$, and multiplied by the time difference between adjacent bins. We start the integration from the local time bin with the first occurrence of 

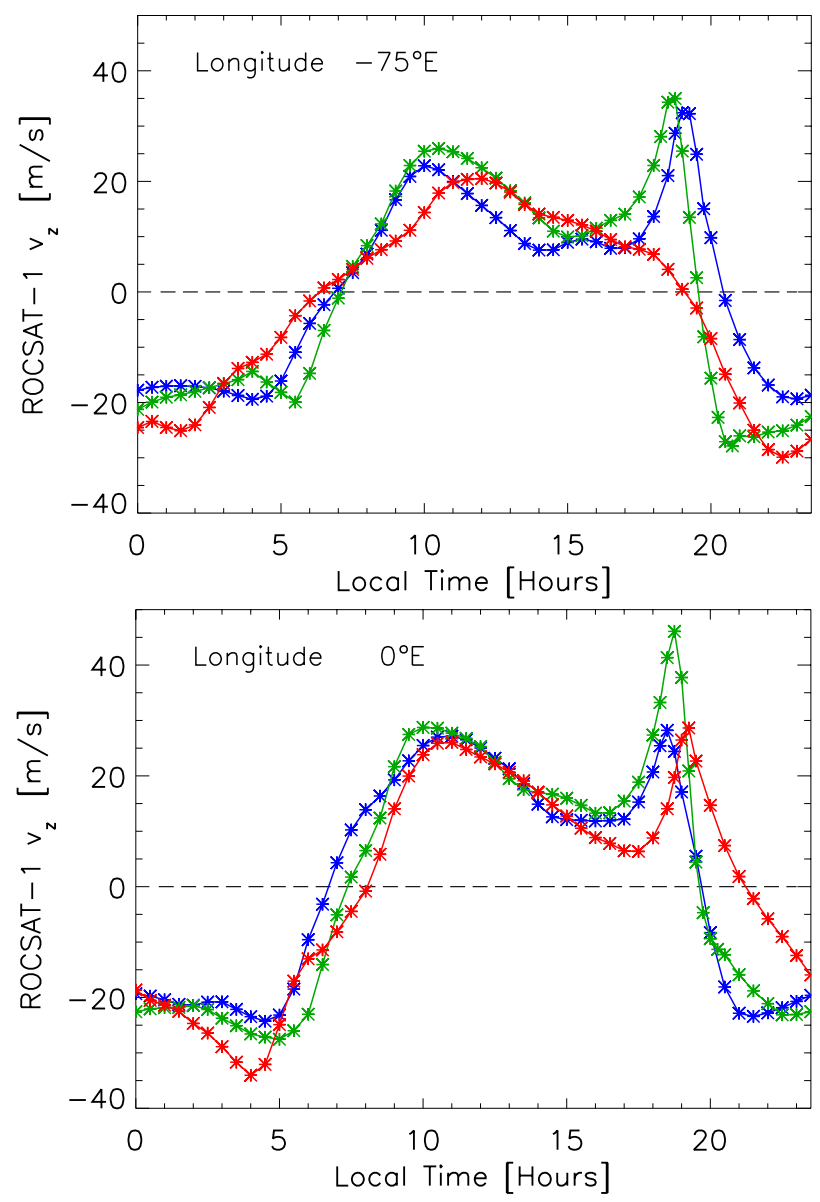

Fig. 1. Local time variation of the equatorial vertical plasma drift at two longitudes, as predicted by the ROCSAT-1 plasma drift model for December solstice (blue), equinox (green), and June solstice (red) for a solar flux level of F10.7=150.

upward plasma drift (positive value of $v_{z}$ ) after the afternoon local minimum (between noon and postsunset maxima) and stop at the last time node before the electric field reverses toward west after the PRE:

$S_{\mathrm{PRE}}=\sum_{i} v_{z, i} \Delta t_{i}$

$S_{\text {PRE }}$ is a measure in length units. If no local minimum could be identified after 16:00 LT (16:30 LT) than the integration started at 17:30 LT (17:15 LT) for solstice seasons (for equinoxes). The slightly different integration interval for equinox was chosen in favour of improvements in correlation between $S_{\text {PRE }}$ and ESF occurrence, as described below.

In Fig. $2 S_{\text {PRE }}$ is plotted versus $v_{z}^{P}$. Both quantities correlate very well with $\mathrm{cc}=0.98$, although the analysis is performed over all longitudes and seasons. The resulting linear regression line has the form, $S_{\mathrm{PRE}}=5.89 v_{z}^{P}-2.91$, where $v_{z}^{P}$ is given in $[\mathrm{m} / \mathrm{s}]$ and $S_{\mathrm{PRE}}$ in $[\mathrm{km}]$. For high values of $v_{z}^{P}$ ( $\sim 50 \mathrm{~m} / \mathrm{s}$, cf. Fig. 2$)$ the plasma rises almost $300 \mathrm{~km}$ during

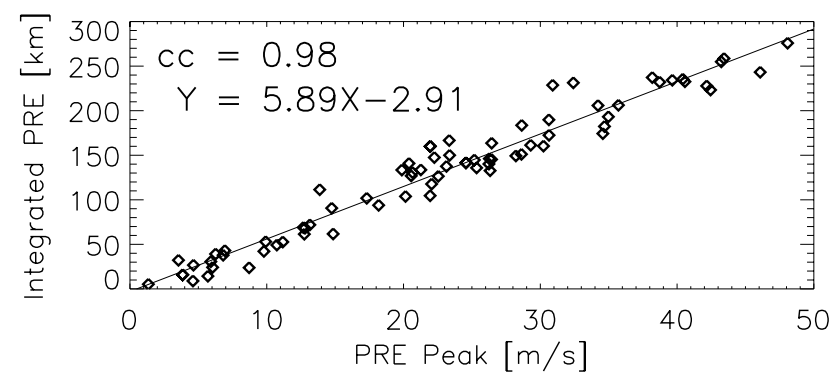

Fig. 2. The integrated plasma drift plotted over the peak plasma drift velocity during the same PRE period for all longitudes and seasons and for a solar flux level of $\mathrm{F} 10.7=150$. Corresponding correlation coefficient (cc) and regression line parameters are provided in the figure.

the PRE lifting phase. The high correlation proves that the peak plasma drift velocity describes well the distance, which the plasma is transported upward over the entire PRE time.

Let us now compare the ESF occurrence with the strength of the PRE. The left panel in Fig. 3 shows the longitudinal variation of the occurrence rate of ESF and of the amplitude of $S_{\mathrm{PRE}}$ for the three seasonal periods. The close relation between both quantities is reflected by high correlation coefficients $(0.90$ for December solstice, 0.84 for equinox months, and 0.90 for June solstice).

The right panel in Fig. 3 provides comparison plots between the ESF occurrence rate and the peak value of the PRE drift velocity, $v_{z}^{P}$. It reveals similar correlation coefficients (0.90 for December solstice, 0.86 for equinox months, and 0.85 for June solstice). The highest correlation is, however, found when the ESF $S_{\text {PRE }}$ curve is shifted a few degrees toward east. Figure 4 shows the cross-correlation coefficients between $S_{\mathrm{PRE}}$ and the ESF occurrence rate for a range of longitude shifts between $-30^{\circ} \mathrm{E}$ and $37.5^{\circ} \mathrm{E}$ in $7.5^{\circ}$ steps. To perform the cross-correlations, the curves of $S_{\mathrm{PRE}}$ and of ESF occurrence rate have been interpolated using a quadratic least square fit. In Fig. 4 the cross-correlation curves are further approximated by a cubic polynomial fit for points between $-7.5^{\circ}$ and $30^{\circ}$. Then we looked for the tangent point of the function. The curve maxima are displaced to the east by $7.8^{\circ}$ $(c c=0.92), 0.7^{\circ}(c c=0.85)$, and $4.9^{\circ}(c c=0.93)$ for December solstice, equinox, and June solstice, respectively.

The linear regression lines between the ESF occurrence rate and $S_{\text {PRE }}$ as derived without eastward shift from their longitudinal variations in Fig. 3 are:

$$
\begin{aligned}
& \mathrm{ESF}_{\mathrm{occ}}=0.21 \cdot S_{\mathrm{PRE}}-8.3 \quad(\mathrm{DS}) \\
& \mathrm{ESF}_{\mathrm{occ}}=0.20 \cdot S_{\mathrm{PRE}}-12.8 \quad(\mathrm{EQ}) \\
& \mathrm{ESF}_{\mathrm{occ}}=0.14 \cdot S_{\mathrm{PRE}}-3.6 \quad(\mathrm{JS})
\end{aligned}
$$

where $S_{\mathrm{PRE}}$ is given in $[\mathrm{km}]$ and the ESF occurrence rate, $\mathrm{ESF}_{\mathrm{occ}}$, in [\%]. The linear regression lines between the ESF occurrence rate and $v_{z}^{p}$ are:

$\mathrm{ESF}_{\mathrm{occ}}=1.33 \cdot v_{z}^{p}-10.0 \quad(\mathrm{DS})$ 

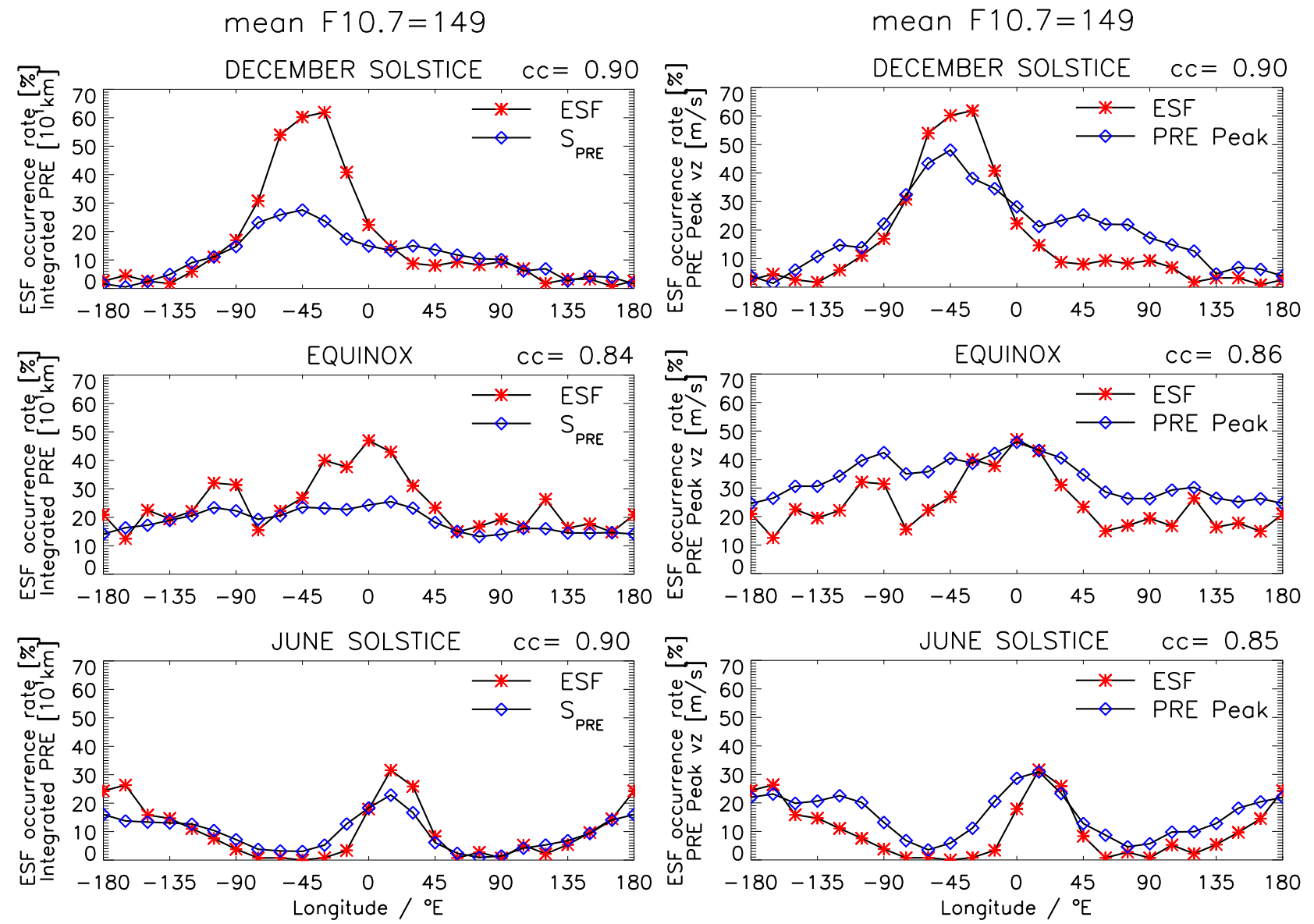

Fig. 3. Longitudinal variation of the occurrence rate of ESF magnetic signatures and integrated PRE plasma drift $S_{\mathrm{PRE}}$ (left panel) and peak plasma drift velocity (right panel) for different seasons.

$$
\begin{aligned}
& \mathrm{ESF}_{\mathrm{occ}}=1.23 \cdot v_{z}^{p}-16.0 \quad(\mathrm{EQ}) \\
& \mathrm{ESF}_{\mathrm{occ}}=1.05 \cdot v_{z}^{p}-6.5 \quad(\mathrm{JS})
\end{aligned}
$$

where $v_{z}^{p}$ is in $[\mathrm{m} / \mathrm{s}]$. Note, these equations describe the globally averaged ESF occurrence rate for each of the seasons.

Equations (3-8) imply that a minimum value of $S_{\mathrm{PRE}}$, or of $v_{z}^{p}$ is required to observe ESF at CHAMP altitude. These global threshold values of $S_{\text {PRE }}$ are $38 \mathrm{~km}, 64 \mathrm{~km}$, and $25 \mathrm{~km}$ for December solstice, equinox and June solstice, respectively. The global threshold $v_{z}^{p}$ are $7.4 \mathrm{~m} / \mathrm{s}, 13.2 \mathrm{~m} / \mathrm{s}$, and $6.2 \mathrm{~m} / \mathrm{s}$. The relations have been calculated for a mean solar flux of F10.7=150. It has been frequently observed that the response of strong ESF to PRE actions depends on solar flux (e.g. Fejer et al., 1999; Manju et al., 2007). We investigated the sensitivity of the threshold PRE actions to the solar flux level. Figure 5 shows the threshold $v_{z}^{p}$ for solar flux levels of F10.7 =100-200 in increments of ten and for each season separately. For better comparison with previously published observations we show the PRE peak velocities rather than $S_{\mathrm{PRE}}$. In this figure, we compared $v_{z}^{p}$ from the ROCSAT1 model at a given F10.7 value with CHAMP-derived ESF occurrence rates within a range of \pm 20 flux units about the center solar flux value. Here we made use of satellite observations from January 2001 to December 2007, to exploit the whole capability of the CHAMP data base. The F10.7 $=150$ values are from Eqs. (6-8). Already at first sight, the threshold $v_{z}^{p}$ increases linearly with solar flux level in all seasons. Figure 5 also includes results from radar observations in India (Jayachandran et al., 1993) and Peru (Fejer et al., 1999), and the observational points have been connected by a dotted line for better visualisation.

We have shown that ESF activity appears preferably in regions with high amplitudes of the PRE, and that there is little ESF occurrences in regions where the PRE is low. In a next step we investigated at which local time sector the ESF occurrence rate responses best to PRE. Therefore, we first examined the local time variation of the PRE peak velocity as a function of season and longitude. This is shown in Fig. 6. The smallest variation is observed during equinoxes, where the PRE reaches its peak at 18:30 LT or 18:45 LT over all longitudes. Larger variations occur during December solstice 


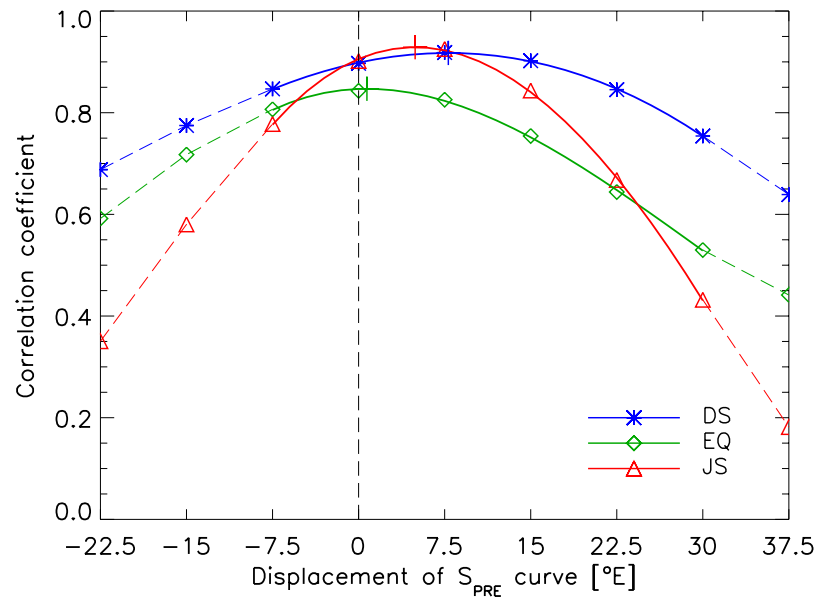

Fig. 4. Cross-correlation between $S_{\mathrm{PRE}}$ and the ESF occurrence rate plotted versus the longitudinal shift of $S_{\mathrm{PRE}}$ location. Peak locations of the approximated functions (solid lines) are marked by vertical dashes

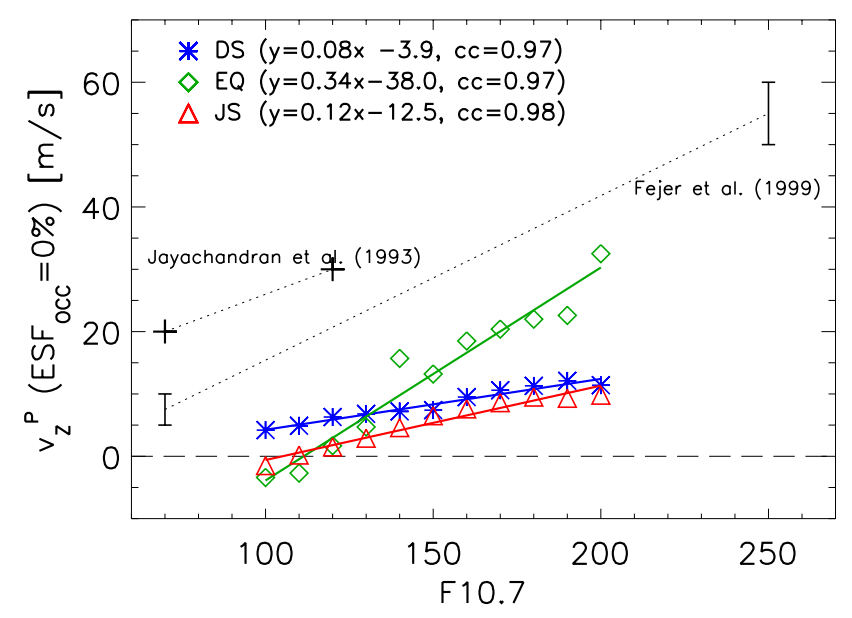

Fig. 5. The threshold $v_{z}^{P}$ plotted versus the solar activity index, F10.7 for different seasons. Results based on radar observations are also included and linked by a dotted line for better visualisation.

with values between 18:15 LT and 19:00 LT. Most significant differences appear during June solstice, where the PRE peak is observed between 18:00 LT and 19:30 LT. It should be noted that in the case of June solstice the indicated local times of the PRE peak at $-90^{\circ},-75^{\circ}$, and $-60^{\circ} \mathrm{E}$ is the time just before the plasma drift velocity starts to decrease rapidly from day to night time values. No typical PRE local maximum of plasma drift developed at these longitudes in this season. Another interesting feature in Fig. 6 is the apparent anti-corellation between the LT-distribution for December and June solstices. For better orientation, the geographic latitudinal variation of the magnetic dip equator is overplotted. For a given longitudinal sector the PRE appears later when the magnetic equator is located in the summer

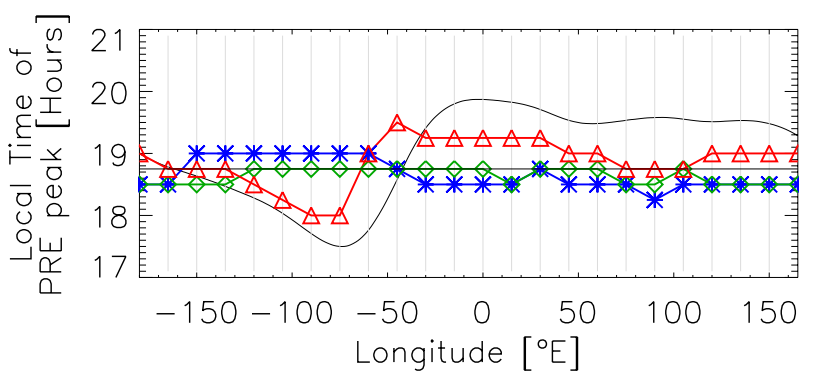

Fig. 6. Local time variation of the occurrence of the PRE plasma drift velocity peak $\left(v_{z}^{P}\right)$ for December Solstice (blue), equinox (green) and June solstice (red) versus longitude. The black curve indicates the latitudinal variation of the dip equator. The black horizontal line represents the geographic equator.

hemisphere than when the magnetic equator is located in the winter hemisphere. The PRE is a result of eastward neutral winds and the sudden decrease of E-region conductivity at sunset (Farley et al., 1986). Figure 6 implies that the PRE's local time is related to the sunset time at the dip equator.

The nearly continuous CHAMP data base allowed for the compilation of a comprehensive map of the s/l distribution of the ESF occurrence rate over local time. Figure 7 shows the ESF occurrence rate for high solar flux years 2001.02004.12 at different local time intervals. The hourly nodes in local time are derived from 2 LT-hours sliding windows of CHAMP observations centered at 19:00 LT for the first and at 03:00 LT for the last node. The occurrence rate of ESF detected by CHAMP increases rapidly betwen 19:00 LT and 20:00 LT. The highest ESF occurrence propabilities is found between 21:00 LT and 23:00 LT in all seasons. The time lag between the PRE occurrence (18:00 LT-19:30LT) and the highest ESF propability corresponds very well with radar observations at Jicamarca (Woodman and LaHoz, 1976; Kudeki and Bhattacharyya, 1999), where bottom side spread- $F$ occurred about $1 \mathrm{~h}$ after sunset and altitude-developed ESF (radar plumes) at about $2-3 \mathrm{~h}$ after sunset. In our statistics mainly altitude-developed ESF are involved since the satellite cannot detect irregularities below its orbital height $(\sim 400 \mathrm{~km})$.

We also performed correlation studies between $S_{\text {PRE }}$ and the ESF occurrence in the different local time sectors to verify how long the correlation is high. Correlation coefficents above 0.6 were revealed until 02:00 LT for all seasons when an eastward shift of $S_{\mathrm{PRE}}$ of $7.5^{\circ} \mathrm{E}$ was considered. Afterwards, the correlation decreased rapidly, e.g. to below 0.2 for June solstice.

\section{Discussion}

We have shown that, as expected, the peak vertical plasma drift velocity of the prereversal enhancement, $v_{z}^{P}$, is highly correlated with the integrated plasma drift velocity, $S_{\mathrm{PRE}}$ 
$(c c=0.98)$, when considering all longitudes and all seasons. Correspondingly, the correlations between $v_{z}^{P}$ and the ESF occurrence rate, and between $S_{\mathrm{PRE}}$ and the ESF occurrence rate are similarly good.

In Fig. 4 we found a longitudinal shift of maximum correlation between the ESF and $S_{\mathrm{PRE}}$ for the solstice month, but the shift was insignificant for equinox. We tested if the eastward propagation of plasma plumes can explain the displacement in solstice. We assume that the ESF event is created at the location of the plasma drift observation around the time of the $v_{z}^{P}, \sim 18: 30 \mathrm{LT}$, and that most of the plumes reach satellite altitudes $(\sim 400 \mathrm{~km})$ about $2 \mathrm{~h}$ later at 20:30 LT. With a typical zonal drifts of ESF of $100 \mathrm{~m} / \mathrm{s}$, we derive a zonal displacement of $6^{\circ}$. This value agrees well with our observations. The significantly lower displacement in equinox suggests the visibility of high bottom side Spread- $F$ which occur earlier than bubbles (Kudeki and Bhattacharyya, 1999). Indeed, the longitudinally averaged $v_{z}^{P}$, and therefore the F layer peak, is higher in equinox, than in solstice months (Fejer et al., 2008), so CHAMP should sample more frequently bottom side Spread- $F$ during equinox.

The correlation between $v_{z}^{P}$ and the ESF occurrence rate is slightly lower than for $S_{\text {PRE }}$, e.g. in June solstice, and it can not be increased by longitudinal shifting. Equation (1) reveals that the ESF growth rate is proportional to the amplitude of the vertical plasma drift velocity (Ott, 1978). The growth rate is furthermore inversely proportional to the ionneutral collision frequency which decreases with increasing height. The integrated PRE velocity combines information from both parameters, hence, it is the preferred indicator for the initiation of ESF.

Here, $S_{\text {PRE }}$ was calculated from a varying integration start time depending on the history of the plasma drift velocity during PRE. However, Haerendel (1973) and Fejer et al. (1999) pointed out that, only the plasma drift after sunset pushes effectively the F-layer profile since ion production terms can be neglegted in lower regions. We also correlated the ESF occurrence rate with $S_{\mathrm{PRE}}$ which was derived from a fixed integration start time at 18:00 LT, the average sunset time. This test resulted in similar, but slightly lower corellation coefficients $(0.88$ for December solstice, 0.84 for equinox months, and 0.83 for June solstice). The lifting of the F-region plasma itself is realised also during day time when signifcant production occurs in all layers (e.g. Stolle et al., 2008). We may assume that upward plasma drift closely before the PRE peak rises the F-region plasma and that such a constellation may favour stronger vertical plasma density gradients at sunset.

A comparable global study was performed by $\mathrm{Su}$ et al. (2008) who correlated occurrence probabilities of plasma irregularities with vertical plasma drift velocities averaged between 18:00 LT and 19:00 LT, both derived from ROCSAT-1 observations. They found similarly high correlation coefficients between 0.70 and 0.91 , except for September equinox (months 8, 9, 10) where the correlation was decreased to

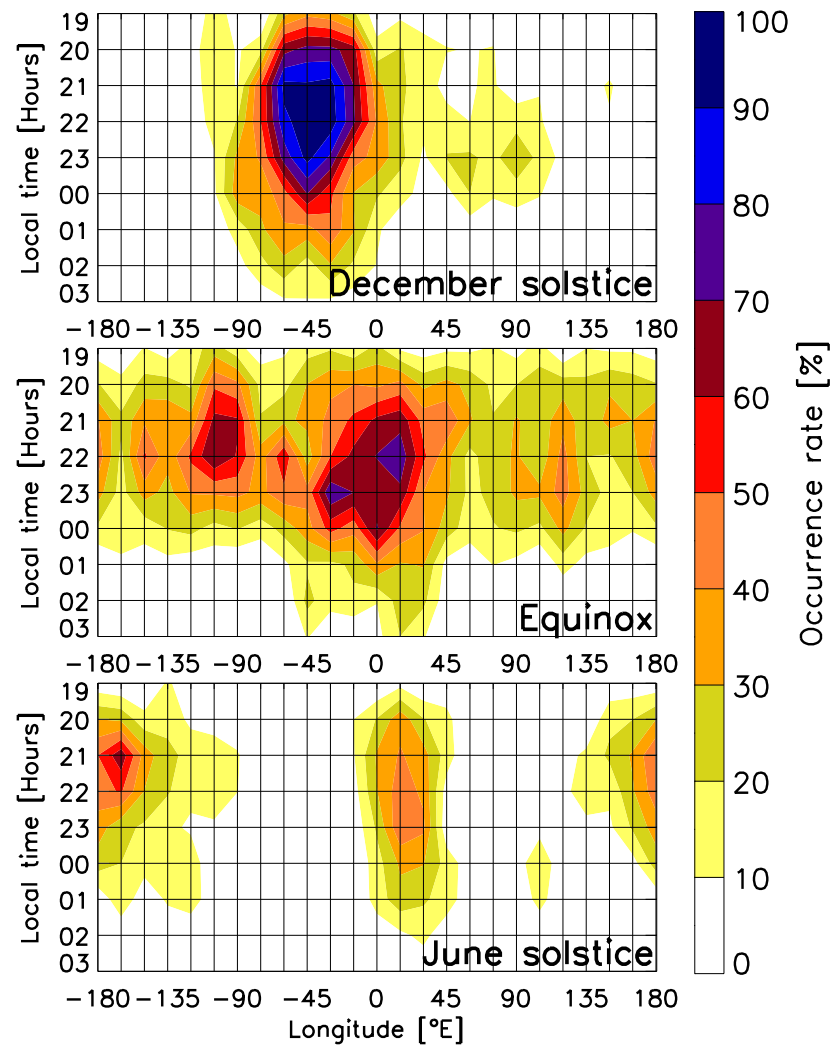

Fig. 7. Longitudinal variations of the occurrence rate of CHAMP ESF signatures for different local times and seasons.

0.14 . They explained this equinoxial differences by different plasma distribution carried on from the preceding solstice season. Also our observations show weakest correlation for the equinox months with 0.85 , but which is still significant. Generaly higher correlations in our study, even from independent data sets, suggest that $v_{z}^{p}$ and $S_{\mathrm{PRE}}$ are the superior indicators for the ESF occurrence over fixed-time averaged plasma drift velocities. Nevertheless, we can support the hypothesis of Su et al. (2008) that the vertical plasma drift during PRE is the most important parameter to determine the $\mathrm{s} / \mathrm{l}$ distribution of large plasma irregularities.

Figure 5 shows the solar flux dependence of the threshold $v_{z}^{p}$ above which we expect ESF occurrence rates higher than $0 \%$ at satellite altitudes. The very high correlation coefficients implies that the required strength of the PRE to initiate strong ESF increases linearly with F10.7, also from global view. The sensitivity seems, however, to be different. While the solstices have similar slopes, the slope for equinox is much higher. This is in contrast to local observations at Jicamarca, where the solar flux sensitivity of $v_{z}^{p}$ is similar in December solstice and equinox (Fejer et al., 1996). However, at Jicamarca, these two seasons have nearly the same $v_{z}^{p}$ as can be seen from Fig. 1, but this is not valid for other longitudes (Fejer et al., 2008). Hence, we neither 
expect such relation from our longitidinal averaged view. A further aspect is the much higher sensitivity of the postsunset ionisation anomaly to solar flux during equinox than during solstice. Liu et al. (2007) found that the crest electron density at CHAMP altitudes increases $0.02-0.025$ times with the solar flux index during equinox while it is only $0.01-0.015$ times during solstices. This may indicate that the PRE drift velocity increases more importantly with solar flux during equinox, therefore also does the treshold $v_{z}^{p}$.

The solstices have similar slopes but with higher threshold $v_{z}^{P}$ during December solstice than during June solstice for all given solar flux levels. It has frequently been observed that the ionosphere and thermosphere are denser during December solstice months than during June solstice months, and this assymetry tends to be enhanced during low solar minimum years (Rishbeth and Müller-Wodarg, 2006). Therefore, we suggest that the resulting increased ion-neutral collision frequency requires higher threshold velocities during December solstice.

For solar flux levels F10.7 $\leq 110$, Fig. 5 shows even negative values for the threshold of $v_{z}^{P}$ for June solstice and equinox. When the solar activity is low, the PRE vertical plasma drift is weak and often downward drifts can occur. Since the plasma density is also weak, some excursions to positive PRE drift values can result in positive R-T growth rates due to the low ion-neutral collision frequency. In our climatological view, this means that the ESF occurrence rate is above zero, even when the mean PRE drift velocity is downward. Few Spread- $F$ events were observed for negative PRE plasma drift values also by Fejer et al. (1999).

We compare retrieved threshold $v_{z}^{P}$ from our analyses with previously published experiments. The generally higher values from local ground-based studies in Fig. 5 imply that we predict some occurrence of ESF even below their thresholds. Here, the different retrieval methods of the threshold should be noted. Our values represent a threshold derived from longitudinal mean plasma drifts and ESF occurrence rates. The radar-based studies show a mean of event-based derived thresholds. Furthermore, the global threshold values stand for an occurrence rate of $0 \%$. This means no ESF event was ever observed under these conditions by the satellite during its multi-years mission time. Therefore, lower threshold values are acceptable.

The nearly continuous CHAMP data base provides maps of the local time variation of the ESF occurrenc rate in different seasons and longitudes which is shown in Fig. 7. One interesting point to investigate is a possible relation between the time of $v_{z}^{P}$ and the onset of significant ESF occurrence in a climatological sense. The largest difference in local time of $v_{z}^{P}$ occurs between the Peruvian $\left(-75^{\circ} \mathrm{E}\right)$ and the Brazilian sector $\left(-45^{\circ}\right.$ E) during June solstice (Fig. 6). However, since the amplitude of plasma velocity itselfs is very low here and there are practically no ESFs observed, this sector is not suitable for investigation. It is also not recommendable to compare the ESF occurrence rates during different seasons at a given longitude since, e.g. December and June solstice show anti-corellated occurrence rates of ESF. As an example, we take the longitude bins $15^{\circ} \mathrm{E}$ during June solstice and $-15^{\circ} \mathrm{E}$ during December solstice since at both longitudes significant ESF occurrence was observed. At these locations the local time of the PRE velocity peak differs by $45 \mathrm{~min}, 19: 15 \mathrm{LT}$ and 18:30LT, for June and December solstice respectively. Correspondingly, Fig. 7 shows the first significant ESF occurrence for June solstice in the 20:00 LT range, but already a considerable amount of ESF events ( $\sim 30 \%$ occurrence rate) was detected around 19:00 LT at $-15^{\circ}$ E during December solstice. From this comparison we may conclude, that the time of the PRE determines the ESF onset, not only on a day-to-day variation, but also in a climatological sense. Also the time of highest occurrence rate is earlier at $-15^{\circ} \mathrm{E}$ during December solstice (21:00 LT-22:00 LT) than at $15^{\circ} \mathrm{E}$ during June solstice (22:00 LT-23:00 LT).

Another important aspect which can be derived from this map is the persistence and temporal evolution of high ESF occurrence propability in different longitude sectors. For example, we compare the American/African longitudes (from $\sim-90^{\circ} \mathrm{E}$ to $45^{\circ} \mathrm{E}$ ) and Pacific longitudes (around $-160^{\circ} \mathrm{E}$ ). In all three seasons, signficant ESF occurrence rates in the American/African sector arise at 20:00 LT, they reach their maximum at 21:00 LT and 22:00 LT, and they decrease after 23:00 LT. Occurrence rates of about 30\% are still observed after local midnight. The ESF occurrence rate in the Pacific sector shows significant values during the equinoxes months and during June solstice. These rapidly increase until 21:00 LT with highest values observed globally at this time, but they decrease quickly after 23:00 LT. Almost no ESFs have been detected after local midnight. Apparently, there are other mechanisms besides the plasma drift during PRE, which are longitudinal dependent that influence the persistence of ESF occurrence. The investigation of such effects would warrant a dedicated study.

\section{Summary}

This study investigated independent climatological data sets of the ESF occurrence rate at $\sim 400 \mathrm{~km}$ altitude obtained from CHAMP magnetic field observations and vertical plasma drift velocities obtained from ROCSAT-1 measurements. We determined the peak vertical plasma drift velocity during PRE, $v_{z}^{p}$, and the integrated vertical drift velocity around PRE, $S_{\mathrm{PRE}}$, as two measures characterising the PRE strength. The longitudinal variation of these PRE quantities were both found to correlate with the ESF occurrence rate to about $90 \%$ in each of the investigated seasons, December solstice, equinox, and June solstice. An even increased correlation was found for the solstices when the ESF occurrence rate was compared to $S_{\mathrm{PRE}}$ shifted eastward by approximately $6^{\circ} \mathrm{E}(0.92$ for December solstice and 0.93 for June solstice). This result indicates that the ESF occurrence rate is closer 
related to $S_{\mathrm{PRE}}$ than to $v_{z}^{p}$. Hence, the uplift in altitude of the plasma is superior in determining large R-T growth rates than the amplitude of the plasma drift velocity itself, also on global scale. The increased correlation with ESF eastward of the $S_{\mathrm{PRE}}$ observation reflects the eastward drift of the ESF events between creation of the irregularity in the lower Fregion and its visibility at satellite altitudes. For Equinox the correlation could rather be increased through displacement, which could be explained by the more frequent detection of bottom side spread- $F$ than plasma bubbles by CHAMP in this season.

The expressions for the linear relation between the PRE and the ESF occurrence rates predict a threshold $v_{z}^{p}$ which increases linearly with solar flux in all seasons. The F10.7 dependence was already found locally (Jayachandran et al., 1993; Fejer et al., 1999), but we showed that this characteristic is valid also in a climatological sense. The global threshold $v_{z}^{p}$ is always higher in December than in June solstice month. We suggest that an increased ion-neutral collision frequency in December requires higher threshold velocities. The highest sensitivity of the threshold drift velocity to F10.7 was found for equinox, which is due to the higher global sensitivity of the evening upward drift to solar flux.

The nearly continuous CHAMP data base allowed for the compilation of a comprehensive map of the s/l distribution of the ESF occurrence rate over local time at $\sim 400 \mathrm{~km}$ and for high solar flux years 2001-2004. This map revealed a longitudinal dependence of the persistence of the plasma irregularities. For example, ESF occurrence rates of about $30 \%$ or more occur in the Atlantic/African sector even after midnight. The occurrence rates in the Pacific sector maximised with high values at 21:00 LT but decreased rapidly afterward, and only low occurrence rates below $10 \%$ exist at midnight. Apparently, there are other mechanisms besides the plasma drift during PRE, which are longitudinal dependent, that influence the persistence of ESF occurrence. It was also found that the local time of significant ESF occurrence rates is later at times and locations where the PRE peak occurs later, not only on day-to-day basis, as was shown in several earlier local studies, but also when climatological data sets are compared.

Acknowledgements. The CHAMP mission is supported by the German Aerospace Center (DLR) in operation and by the Federal Ministry of Education (BMBF), as part of the Geotechnology Programme, in data processing. The ROCSAT-1 data is processed under grant 93-NSPO(B)-IPEI-FA07-01 from the National Space Organization of the Republic of China.

Topical Editor M. Pinnock thanks two anonymous referees for their help in evaluating this paper.

\section{References}

Abdu, M. A., Sobral, J. H. A., and Batista, I. S.: Equatorial spreadF statistics in the American longitudes: some problems relevant to ESF description in the IRI scheme, Adv. Space Res., 25, 113124, 2000.

Burke, W. J., Gentile, L. C., Huang, C. Y., Valladares, C. E., and Su, S. Y.: Longitudinal variability of equatorial plasma bubbles observed by DMSP and ROCSAT-1, J. Geophys. Res., 41, A12301, doi:10.1029/2004JA010583, 2004.

Farley, D. Balsey, B., Woodman, R., and McClure, J.: Equatorial Spread F: Implications of VHF Radar Observations, J. Geophys. Res., 75, 7199-7216, 1970.

Farley, D. T., Bonelli, E., Fejer, B. G., and Larsen, M. F.: The prereversal enhancement of the zonal electric field in the equatorial ionosphere, J. Geophys. Res., 91, 13 723-13 728, 1986.

Fejer, B. G., de Paula, E. R., Scherliess, L., and Batista, I. S.: Incoherent scatter radar, ionosonde and satellite measurements of equatorial $\mathrm{F}$ region vertical plasma drifts in the evening sector, Geophys. Res. Lett., 23, 1733-1736, 1996.

Fejer, B. G., Scherliess, L., and de Paula, E. R.: Effect of the vertical plasma drift velocity on the generation and evolution of equatorial spread F, J. Geophys. Res., 104, 19 859-19 869, 1999.

Fejer, B. G., Jensen, J. W., and Su, S.-Y.: Quiet-time equatorial $F$ region vertical plasma drift model derived from ROCSAT1 observations, J. Geophys. Res., 113, A05304, doi:10.1029/ 2007JA012801, 2008.

Haerendel, G.: Theory of equatorial spread F, in: Report des MPI fuer extraterrestrische Physik, unpublished manuscript, 1973.

Huang, C. Y., Burke, W. J., Machuzak, J. S., Gentile, L. C., and Sultan, P.: DMSP observations of equatorial plasma bubbles in the topside ionosphere near solar maximum, J. Geophys. Res., 106, 8131-8142, 2001.

Jayachandran, B., Balan, N., Rao, P. B., Sastri, J. H., and Bailey, G. J.: HF Doppler and ionosonde observations on the onset conditions of equatorial spread- $F$, J. Geophys. Res., 98, 13741$13750,1993$.

Kelley, M. C.: The Earth's Ionosphere: Plasma Physics and Electrodynamic, Academic Press, Inc. San Diego, 1989.

Kil, H. and Heelis, R. A.: Global distribution of density irregularities in the equatorial ionosphere, J. Geophys. Res., 103, 407-417, 1998.

Kudeki, E. and Bhattacharyya, S.: Postsunset vortex in equatorial $F$-region plasma drifts and implication for botomside spread- $F$, J. Geophys. Res., 104, 28 163-28 170, 1999.

Lin, S. L., Immel, J. T., Yeh, H.-C., Mende, S. B., and Burch, J. L.: Simultaneous observations of equatorial plasma depletion by IMAGE and ROCSAT-1 satellites, J. Geophys. Res., 110, A06304, doi:10.1029/2004JA010774, 2005.

Liu, H., Stolle, C., Förster, M., and Watanabe, S.: Solar activity dependence of the electron density at $400 \mathrm{~km}$ at equatorial and low latitudes observed by CHAMP, J. Geophys. Res., 112, A11311, doi:10.1029/2007JA012616, 2007.

Makela, J. J. and Kelley, M. C.: Field-aligned 777.4-nm composite airglow images of equatorial plasma depletions, Geophys. Res. Lett., 30, 1442, doi:10.1029/2003GL017106, 2003.

Manju, G., Devasia, C. V., and Sridharan, R.: On the seasonal variation of the treshold height for occurrence of equatorial spread $\mathrm{F}$ during solar minimum and maximum years, Ann. Geophys., 25, 855-861, 2007, 
http://www.ann-geophys.net/25/855/2007/.

Maruyama, T., Nozaki, K., Yamamoto, M., and Fukao, S.: Ionospheric height changes at two closely separated equatorial stations and implications in spread $F$ onset, J. Atmos. Solar-Terr. Phys., 64, 1557-1563, 2002.

Maus, S., Rother, M., Stolle, C., Mai, W., Choi, S., Lhr, H., Cooke, D., and Roth, C.: Third generation of the Potsdam Magnetic Model of the earth (POMME), Geochem. Geophys. Geosyst., 7, Q07008, doi:10.1029/2006GC001269, 2006.

McClure, J. P., Singh, S., Bamgboye, D. K., Johnson, F. S., and Kil, H.: Occurrence of equatorial $F$ region irregularities: Evidence for tropospheric seeding, J. Geophys. Res., 103, 29 119-29 135, 1998.

Mendillo, M., Meriwether, J., and Biondi, M.: Testing the thermospheric wind supression mechanism for day-to-day variability of equatorial spread-F, J. Geophys. Res., 106, 3655-3663, 2001.

Nishioka, M., Saito, A., and Tsugawa, T.: Occurrence characteristics of polasma bubbles derived from global ground-based GPS receiver networks, J. Geophys. Res., 113, A05301, doi: 10.1029/2007JA012605, 2008.

Ossakow, S. L.: Spread $F$ theories - A review, J. Atmos. Solar-Terr. Phys., 43, 437-452, 1981.

Ott, P.: Theory of Rayleigh-Taylor bubbles in the equatorial ionosphere, J. Geophys. Res., 83, 2066-2070, 1978.

Palmroth, M., Lassko, H., Fejer, B. G., and Pfaff Jr., R. F.: DE 2 observations of morningside and eveningside plasma density depletions in the equatorial ionosphere, J. Geophys. Res., 105, 18 429-18 442, 2001.

Park, J., Min, K. W., Kim, V. P., H., K., Lee, J.-J., Kim, H.J., Lee, E., and Lee, D. Y.: Global distribution of equatorial plasma bubbles in the premidnight sector during solar maximum as observed by KOMPSAT-1 and Defense Meteorological Satellite Program F15, J. Geophys. Res., 110, A07308, doi: 10.1029/2004JA010817, 2005.

Park, S. H., England, S. L., Immel, T. J., Frey, H. U., and Mende, S. B.: A method for determining the drift velocity plasma depletions in the equatorial ionosphere using far-ultraviolet spacecraft observations., J. Geophys. Res., 112, A11314, doi:10.1029/ 2007JA012327, 2007.

Pimenta, A. A., A., B. J., Fagundes, P. R., Sahai, Y., Buriti, R. A., Takahashi, H., and Taylor, M. J.: Ionospheric plasma bubble zonal drifts over the tropical region: a study using OI $630 \mathrm{~nm}$ emission all-sky images, J. Atmos. Solar-Terr. Phys., 65, $1117-$ 1126, 2003.

Reigber, C., Lühr, H., and Schwintzer, P.: CHAMP mission status, Adv. Space Res., 30, 129-134, 2002.
Rishbeth, H. and Müller-Wodarg, I. C. F.: Why is there more ionosphere in January than in July? The annual assymetry of the F-layer, Ann. Geophys., 24, 3293-3311, 2006, http://www.ann-geophys.net/24/3293/2006/.

Sahai, Y., Fagundes, P. R., Abalde, J. R., Pimenta, J. A., Otsuka, Y., and Rios, V. H.: Generation of large-sacle equatorial F-region plasma depletions during low range spread-F season, Ann. Geophys., 22, 15-23, 2004, http://www.ann-geophys.net/22/15/2004/.

Sobral, J. H. A., Abdu, M. A., Takahashi, H., Sawant, H., Zamlutti, C. J., and Borba, G. L.: Solar and geomagnetic activity effects on nocturnal zonal velocities of ionospheric plasma depletions, Adv. Space Res., 24, 1507-1510, 1999.

Stolle, C., Lühr, H., Rother, M., and Balasis, G.: Magnetic signatures of equatorial spread $\mathrm{F}$, as observed by the CHAMP satellite, J. Geophys. Res., 111, A02304, doi:1029/2005JA011184, 2006.

Stolle, C., Manoj, C., Lühr, H., Maus, S., and Alken, P.: Estimating the day time Equatorial Ionization Anomaly strength from electric field proxies, J. Geophys. Res., 113, A09310, doi: 10.1029/2007JA012781, 2008.

Su, S.-Y., Chao, C. K., and Liu, C. H.: On monthly/seasonal/longitidinal variations of equatorial irregularitiey occurrences and their relationship with the post-sunset vertical drift velocity, J. Geophys. Res., 113, A05307, doi: 10.1029/2007JA012809, 2008.

Sultan, P. J.: Linear theory and modeling of the Rayleigh-Taylor instability leading to the occurrence of equatorial spread $F$, J. Geophys. Res., 101, 26 875-26891, 1996.

Tsunoda, R. T.: Control of the seasonal and longitudinal occurrence of equatorial scintillations by the longitudinal gradient in integrated $E$ region Pedersen Conductivity, J. Geophys. Res., 90, 447-456, 1985.

Whalen, J. A.: An equatorial bubble: Its evolution observed in relation to bottomside spread $\mathrm{F}$ and to the Appleton anomaly, J. Geophys. Res., 105, 5303-5315, 2000.

Wiens, R. H., Ledvina, B. M., Kintner, P. M., Afewerki, M., and Mulugheta, Z.: Equatorial plasma bubbles in the ionosphere over Eritrea: occurrence and drift speed, Ann. Geophys., 24, 14431453, 2006, http://www.ann-geophys.net/24/1443/2006/.

Woodman, R. F. and LaHoz, C.: Radar observations of F-region equatorial irregularities, J. Geophys. Res., 81, 5447-5466, 1976.

Yao, D. and Makela, J. J.: Analysis of equatorial plasma bubble zonal drift velocites in the Pacific sector by imaging techniques, Ann. Geophys., 25, 701-709, 2007, http://www.ann-geophys.net/25/701/2007/.

Yeh, H.-C., Su, S. Y., Yeh, Y. C., Wu, J. M., Heelis, R. A., and Holt, B. J.: Scientific mission of the IPEI payload onboard ROCSAT-1, Terr. Atmos. Ocean. Sci., supplementary issue, 19-42, 1999. 